\title{
Front Matter: Volume 10448
}

, "Front Matter: Volume 10448," Proc. SPIE 10448, Optifab 2017, 1044801 (7 December 2017); doi: 10.1117/12.2305130

SPIE. Event: SPIE Optifab, 2017, Rochester, New York, United States 


\section{PROCEEDINGS OF SPIE}

\section{Optifab 2017}

Julie L. Bentley

Sebastian Stoebenau

Editors

\section{6-20 October 2017 \\ Rochester, New York, United States}

Sponsored by

SPIE

Cosponsored by

The American Precision Optics Manufacturers Association (United States)

Published by

SPIE 
The papers in this volume were part of the technical conference cited on the cover and title page. Papers were selected and subject to review by the editors and conference program committee. Some conference presentations may not be available for publication. Additional papers and presentation recordings may be available online in the SPIE Digital Libra ry at SPIEDigita lLibra ry.org.

The papers reflect the work and thoughts of the authors and a re published herein as submitted. The publisher is not responsible for the validity of the infomation or for any outcomes resulting from reliance thereon.

Please use the following format to cite material from these proceedings:

Author(s), "Title of Paper," in Optifa b 2017, edited by J ulie L. Bentley, Sebastian Stoebenau, Proceedings of SPIE Vol. 10448 (SPIE, Bellingham, WA, 2017) Seven-digit Article CID Number.

ISSN: 0277-786X

ISSN: 1996-756X (electronic)

ISBN: 9781510613645

ISBN: 9781510613652 (elec tronic)

Published by

SPIE

P.O. Box 10, Bellingham, Washington 98227-0010 USA

Telephone +1 3606763290 (Pacific Time)· Fax +1 3606471445

SPIE.org

Copyright $\odot 2017$, Society of Photo-Optic al Instrumentation Engineers.

Copying of material in this book for intemal or personal use, or for the intemal or personal use of specific clients, beyond the fair use provisions granted by the U.S. Copyright Law is authorized by SPIE subject to payment of copying fees. The Transactional Reporting Service base fee for this volume is $\$ 18.00$ per a rticle (or portion thereof), which should be paid directly to the Copyright Clearance Center (CCC), 222 Rosewood Drive, Danvers, MA 01923. Payment may also be made electronically through CCC Online at copyright.com. Other copying for republic ation, resale, a dvertising or promotion, or any form of systematic or multiple reproduction of any material in this book is prohibited except with pemission in writing from the publisher. The CCC fee code is 0277$786 \mathrm{X} / 17 / \$ 18.00$.

Printed in the United States of America.

Publication of record for individual papers is online in the SPIE Digital Library.

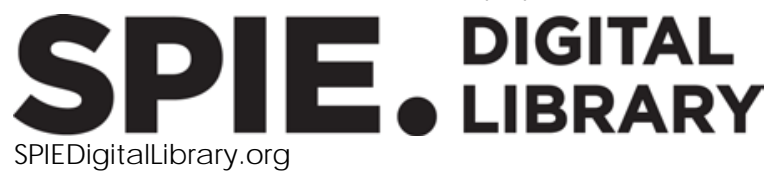

Paper Numbering: Proceedings of SPIE follow an e-First publication model. A unique citation identifier (CID) number is assigned to each article at the time of publication. Utilization of CIDs allows articles to be fully citable as soon as they are published online, and connects the same identifier to all online and print versions of the publication. SPIE uses a seven-digit CID article numbering system struc tured as follows:

- The first five digits correspond to the SPIE volume number.

- The last two digits indic ate public ation order within the volume using a Base 36 numbering system employing both numerals and letters. These two-number sets start with $00,01,02,03,04$, 05, 06, 07, 08, 09, 0A, OB ... 0Z, followed by 10-1Z, 20-2Z, etc. The CID Number appears on each page of the manuscript. 


\title{
Contents
}

\author{
ix Authors \\ xiii Conference Committee
}

\section{PLENARY SESSION}

1044802 Freeform Optics: current challenges for future serial production (Plenary Paper) [10448-25]

1044803 Concept for a new approach to realize complex optical systems in high volume (Plenary Paper) [10448-26]

\section{GRINDING AND POLISHING PROCESSES I}

1044804 Analysis and optimization of surface profile correcting mechanism of the pitch lap in largeaperture annular polishing [10448-1]

1044805 APS 3D: a new benchmark in aspherical polishing [10448-2]

1044806 Novel high-NA MRF toolpath supports production of concave hemispheres [10448-3]

$1044807 \quad$ Ultrasonic grinding of optical materials [10448-4]

1044809 Etching hard brittle optical materials by masked ion beam [10448-6]

\section{GRINDING AND POLISHING PROCESSES II}

10448 OA New surface smoothing technologies for manufacturing of complex shaped glass components [10448-7]

10448 OE The broad utility of Trizact diamond tile [10448-11]

$10448 \mathrm{OF}$ Impact of slurry $\mathrm{pH}$ on material removal rate and surface quality of polished fused silica [10448-12]

\section{GRINDING AND POLISHING PROCESSES III}

$10448 \mathrm{OH}$ New high-precision deep concave optical surface manufacturing capability [10448-14]

10448 Ol Precision production: enabling deterministic throughput for precision aspheres with MRF [10448-15]

10448 0J Evolving rocket optics applications drive manufacturing advances [10448-16] 
10448 OK Applying MRF to errors caused by optical and opto-mechanical assembly [10448-17]

$10448 \mathrm{OL} \quad$ Novel process for production of micro lenses with increased centering accuracy and imaging performance [10448-18]

\section{ADDITIVE MANUFACTURING}

$10448 \mathrm{ON}$ Simple scattering analysis and simulation of optical components created by additive manufacturing [10448-20]

10448 OP Current use and potential of additive manufacturing for optical applications [10448-22]

\section{OPTICAL DESIGN AND ENGINEERING}

10448 OR Tolerancing aspheres based on manufacturing knowledge [10448-28]

10448 OS The importance of understanding manufacturing distributions in simulating manufactured performance of optical systems [10448-29]

10448 OT Integrating optical, mechanical, and test software (with applications to freeform optics) [10448-30]

10448 OV Twyman effects in thin curved optics [10448-32]

\section{DIAMOND TURNING}

10448 OW Analysis of the application of poly-nanocrystalline diamond tools for ultra precision machining of steel with ultrasonic assistance [10448-33]

10448 OX Micro-laser assisted machining: the future of manufacturing silicon optics [10448-34]

10448 OY UPC 300 ultra precise fast tool freeform machining system with integrated metrology for corrective machining [10448-35]

\section{FREEFORM FABRICATION AND TESTING}

1044813 Fabrication and correction of freeform surface based on Zernike polynomials by slow tool servo [10448-42]

1044814 Precision asphere and freeform optics manufacturing using plasma jet machining technology [10448-43]

1044815 Computer aided manufacturing for complex freeform optics [10448-44]

1044816 Shape measurement of freeform surfaces using experimental ray tracing [10448-45]

1044817 Metrology for the manufacturing of freeform optics [10448-46] 
1044818 Enhanced resolution and accuracy of freeform metrology through Subaperture Stitching Interferometry [10448-47]

\section{METROLOGY I}

1044819 From optics testing to micro optics testing [10448-48]

$104481 \mathrm{~A} \quad$ Asphere cross testing: an exercise in uncertainty estimation [10448-49]

10448 1B Advancements in non-contact metrology of asphere and diffractive optics [10448-50]

10448 1C Spectrally controlled interferometry for measurements of flat and spherical optics [10448-51]

10448 1D Surface characterization protocol for precision aspheric optics [10448-52]

\section{METROLOGY II}

$104481 \mathrm{E} \quad$ SUN: A fully automated interferometric test bench aimed at measuring photolithographic grade lenses with a sub nanometer accuracy [10448-53]

$104481 G \quad$ Centering steep aspheric surfaces [10448-55]

$104481 \mathrm{H} \quad$ Automated asphere centration testing with AspheroCheck UP [10448-56]

METROLOGY III

$1044811 \quad$ Tailored complex degree of mutual coherence for plane-of-interest interferometry with reduced measurement uncertainty [10448-57]

$104481 \mathrm{~J} \quad$ Absolute surface form measurement of large flat optics based on oblique incidence method [10448-58]

\section{OPTICAL MATERIALS}

$104481 \mathrm{~L} \quad$ Stability requirements for two-beam interference lithography diffraction grating manufacturing [10448-60]

$104481 \mathrm{M}$ Thermal instability of BK7 and how it affects the manufacturing of large high precision surfaces [10448-61]

$104481 \mathrm{~N}$ Commercializing potassium terbium fluoride, $\mathrm{KTF}\left(\mathrm{KTb}_{3} \mathrm{~F}_{10}\right)$ faraday crystals for high laser power optical isolator applications [10448-62]

$1044810 \quad$ UV-cured polymer optics [10448-63] 
10448 IP Brilluoin spectroscopy application for express, non-contact testing of glass and polymer products [10448-64]

10448 1Q Application of speckle shearing interferometry to the evaluation of creep strain in elastomers [10448-65]

\section{COATING AND CLEANING}

10448 is Rare earth-based low-index films for IR and multispectral thin film solutions [10448-67]

10448 1T Prospects for the enhancement of PIAD processes by plasma diagnostics [10448-69]

10448 IV Multilayer coating of optical substrates by ion beam sputtering [10448-71]

\section{POSTER SESSION}

10448 IW Non-conventional optomechanical choppers: analysis and design of novel prototypes [10448-72]

10448 IY High precision processing $\mathrm{CaF}_{2}$ application research based on the magnetorheological finishing (MRF) technology [10448-75]

10448 iz An optimized method to calculate error correction capability of tool influence function in frequency domain [10448-76]

1044821 Precision lens assembly with alignment turning system [10448-78]

1044822 Cheap and fast measuring roughness on big surfaces with an imprint method [10448-79]

1044823 The study of sub-surface damage distributions during grinding process on different abrasion materials [10448-80]

1044824 Manufacturing of three dimensional silicate moldings by selective laser beam sintering [10448-81]

1044825 Optical characterisation of hydroxide catalysed bonds applied to phosphate glass [10448-82]

1044828 Newly patented process enables low-cost solution for increasing white light spectrum of LEDs [10448-85]

1044829 Enhanced measuring range with aspheric transmission spheres [10448-86]

10448 2B Glass molding of 3mm diameter aspheric plano-convex lens [10448-88]

10448 2D Measurement of strongly curved surfaces by multi-beam experimental ray tracing [10448-90]

$104482 F \quad$ Freeform optics manufacturing [10448-92] 
$104482 \mathrm{G} \quad$ Fabrication of advanced glass light pipes for solar concentrators (Best Student Paper Award) [10448-93]

$104482 \mathrm{H} \quad$ Study on a magneto-rheological removal process of periodic turning marks [10448-95]

$1044821 \quad$ Material of LAPAN's thermal IR camera equipped with two microbolometers in one aperture [10448-96]

10448 2J Design of a solar concentrator considering arbitrary surfaces (Runner-Up Best Student Paper Award) [10448-97]

10448 2L Laser scattering technique to characterize turbulent liquid [10448-100]

$104482 \mathrm{M}$ Spectroscopic enhancement study in $\mathrm{Yb}^{3+} / \mathrm{Er}^{3+}$ doped ferroelectric $\mathrm{SrTiO}_{3}$ ceramics [10448-101]

$104482 \mathrm{~N} \quad$ An efficient way to fabricate micro transmission grating inside quartz and PDMS material by femtosecond laser micromachining [10448-102]

$104482 Q \quad$ Design of an ultra-precision CNC chemical mechanical polishing machine and its implementation [10448-105]

$104482 R \quad$ Optical designs for MWIR and four quadrant detectors by using beam steering methods in missile applications [10448-106]

1044825 Four and eight faceted domes effects on drag force and image in missile application [10448-107] 
Proc. of SPIE Vol. 10448 1044801-8

Downloaded From: https://www.spiedigitallibrary.org/conference-proceedings-of-spie on 26 Apr 2023 Terms of Use: https://www.spiedigitallibrary.org/terms-of-use 


\section{Authors}

Numbers in the index correspond to the last two digits of the seven-digit citation identifier (CID) article numbering system used in Proceedings of SPIE. The first five digits reflect the volume number. Base 36 numbering is employed for the last two digits and indicates the order of articles within the volume. Numbers start with 00, 01, 02, 03, 04, 05, 06, 07, 08, 09, 0A, 0B...0Z, followed by 10-1Z, 20-2Z, etc.

\author{
A., Bustanul, 21 \\ Arnold, Thomas, 14 \\ Atkins, R., 2G \\ Avendaño-Alejo, Maximino, 2J \\ Bai, Yunbo, $1 \mathrm{~J}$ \\ Ban, Xinxing, $2 Q$ \\ Barragán-Pérez, Omar, 1Q \\ Bartlett, Kevin, 0J \\ Bechtold, Michael, 07 \\ Bechtold, Rob, 07 \\ Binkele, T., 16 \\ Blalock, Todd, 17 \\ Bliedtner, Jens, 0A, 24 \\ Boehm, Georg, 14 \\ Bouafia, Mohamed, 2L \\ Bourgois, R., $1 \mathrm{E}$ \\ Brinkmann, Ralf P., IT \\ Brock, Christian, 19 \\ Bruder, A., 24 \\ Brunelle, Matthew, OP, 17, 1M \\ Bulla, B., OW \\ Cahill, Michael, 07 \\ Chang, Keng-Shou, 23 \\ Chen, Fong-Zhi, 21 \\ Cheng, Yuan-Chieh, 13 \\ Cira, Octavian, $1 \mathrm{~W}$ \\ Cramer, Neil, 10 \\ Csukas, Eduard Sebastian, IW \\ Dambon, O., OW \\ Daniel, M. V., IV \\ Das, U., 2N \\ Davenport, Amelia, 10 \\ DeFisher, Scott, 1B \\ DeGroote Nelson, Jessica, oJ \\ DeMarco, Mike, OK \\ Demian, Dorin, $1 \mathrm{~W}$ \\ Demmler, M., IV \\ Díaz-Uribe, Rufino, 2J \\ Doetz, M., OW \\ Dogan, Y., 2G \\ Donohue, Keith, $\mathrm{OH}$ \\ Doodala, Somaiah, ID \\ Dorn, Ralf, 19 \\ Duma, Virgil-Florin, $1 \mathrm{~W}$ \\ Dumas, Paul, 06, OH, Ol, 18 \\ Entezarian, Navid, $\mathrm{Ol}$ \\ Fan, Bin, $1 Y$ \\ Ferralli, Ian, OP, 17 \\ Fess, Edward, 07, 15
}

Fleischmann, F., 16, 2D

Foest, Rüdiger, $1 T$

Foundos, Greg, $1 \mathrm{~N}$

Franke, Christian, IT

Frisch, Greg, 1M

Fu, Taotao, 09

Fuchs, U., OR, 29

Fuhr, Michael, OA

Fütterer, G., 11

Gagliardi, John I., OE

García-Díaz, Reyes, 2J

Gauch, Daniel, 05, OY

Gemballa, Jake, 2F

Genberg, Victor, OT

Gerhardt, Martin, OA

Ghar, Amar, 2N

Glaser, Tilman, $1 \mathrm{~L}$

Götze, Kerstin, 0A, 24

Gregory, Michael, 1M

Grüger, Heinrich, 03

Gu, Yawen, $2 Q$

Hahne, F., $1 \mathrm{H}$

Hall, Christopher, OK

Hamy, A. L., $1 \mathrm{E}$

Han, Jeong-Yeol, $2 \mathrm{H}$

Harhausen, Jens, IT

Heinrich, A., ON

Henkel, Sebastian, OA

Henning, T., 16, 2D

Hilbig, David, 16, 2D

Ho, Cheng-Fang, 21

Horsak, A., ON

Hou, Xi, 1 Z

Hough, James, 25

Hsu, Ming-Ying, 13

Hsu, Wei-Yao, 13, 21, 23

$\mathrm{Hu}, \mathrm{C} ., 2 \mathrm{G}$

Huang, Chien-Yao, 21, 23

Hue, Myung sang, 2B

Huttenhuis, Stephan, OY

Hyman, Michael, 1M

Hyun, Sang-Won, $2 \mathrm{H}$

Jeon, Min-Woo, $2 \mathrm{H}$

Jeong, Byeong-Joon, $2 \mathrm{H}$

Jia, Xin, 09

Jiang, Chunye, $2 Q$

Jiménez-Rodríguez, Martín, 2J

Johns, Dustin, 15

Killow, Christian J., 25 
Kim, Dongguk, 2B

Kim, Geon-Hee, 2H

Klocke, F., OW

Knobbe, Jens, 03

Koch, Felix, 1L

Kode, Sai Kumar, OX

Köhler, T., 02

Kokot, S., OR

Kotaria, Rajendra, 1D

Kumar, Kaushal, 2M

Kuo, Hui-Jean, 21

Kuo, Ching-Hsiang, 21, 23

Lacaille, Grégoire, 25

Lakhal, Malika, 2L

Lambropoulos, John C., OV

Langehanenberg, P., OL, $1 \mathrm{H}$

Lee, Giljae, 2B

Lee, Kye-Sung, $2 \mathrm{H}$

Lehr, Dennis, $1 \mathrm{~L}$

LePage, Gabriel, 15

Leuckefeld, Michael, 03

Li, Yun, 09

Liebl, J., 22

Lin, Yi-Hao, 21

Liu, Shijie, $1 \mathrm{~J}$

Logunov, Stephan L., IP

Lynch, Tim, 17

MacKay, Peter E., 25

Madsen, C. K., $2 \mathrm{G}$

Maloney, Chris, 06, OH, 0I, 18

Manallah, Aïssa, 2L

Mangano, Valentina, 25

Martínez-Enríquez, Arturo I., 2J

Matthews, Greg, 15, 2F

Maunier, Cedric, OF

Medicus, Kate, OP

Men, Shi, $1 \mathrm{~J}$

Messner, Bill, OK

Meyer, Sebastian, 03

Michels, Gregory, OT

Michtchenko, Alexandre, 1Q

Mikulic, Dalibor, 05

Mitchell, Mike, OX

Möhl, A., 29

Morrison, Don, OX

Morrison, M., 2G

Murphy, Paul E., 18, 1A

Myer, Brian, 0J, OT, 17

Neauport, Jerome, OF

Neff, Joe, is

Nestler, Matthias, OK

Niehaus, Frank, OY

Notargiacomo, Mark, IM

Oberberg, Moritz, IT

Olszak, Artur G., 1C

Ortiz-Gonzáles, Antonio de Jesús, 1Q

P., Inwan, 2I

Paetzelt, Hendrik, 14

Panigrahi, P. K., 2N

Panwar, Rakesh S., ID
Parks, Robert E., $1 G$

Pascual-Francisco, Juan Benito, 1Q

Payne, Alexis, $1 \mathrm{~N}$

Peng, Wei-Jei, 13

Perdue, Jamie, 0J

Pfund, Johannes, 19

Piché, François, $\mathrm{OH}$

Piñón, Victor, III, 10

Poliakoff-Leriche, Karine, OF

Pop, Nicolina, IW

Pourcelot, P., 1E

Pügner, Tino, 03

Rädlein, Edda, OA

Rank, M., ON

Rascher, R., 22

Ravindra, Deepak, OX

Redien, Melanie, OF

Reinig, Peter, 03

Remy, Bertrand, OF

Robertson, D. J., OW

Romero, Vincent D., OE

Ross, James, $2 \mathrm{~F}$

Roth, E., 02

Rowan, Sheila, 25

Ryu, Geunman, 2B

Sakarya, Doğan Uğur, 2R, 2S

Sakthibalan, Siva, 1D

Salsbury, Chase, 1C

Sanson, Mark C., OS

Santiago, Freddie, 10

Sanyogita, $2 \mathrm{~N}$

Sarepaka, RamaGopal V., ID

Schenk, T., OL

Schindler, C., 02

Schlichting, Wolfgang, iN

Schluntz, Nohl, 1M

Schopf, C., 22

Schottka, K., OW

Schulze, Christian, OA

Schulze, Jan, 2D

Schwager, Anne-Marie, OA, 24

Shao, Jianda, 04, 1J

Shi, Chunyan, 12

Shu, Shyu-Cheng, 23

Solmaz, M., 2G

Spanard, Jan-Marie, 28

Stenzel, Olaf, IT

Stephan, Thomas, 07

Stevens, Kevin, 1N

Stolze, Markus, is

Stroshine, Chris, OX

Sukul, Prasenjit Prasad, 2M

Sung, Hayeong, 2B

Supranowitz, Chris, 06, OH, 18

Susarrey-Huerta, Orlando, 1Q

Sventek, Bruce, OE

T., Andi M., 2 l

Triftshauser, Jeremiah, $1 M$

van Veggel, Anna-Maria A., 25

VanKerkhove, Steve, $\mathrm{OH}$ 
Vassmer, D., 16

Veit, Christian, 05

Verduzco-Grajeda, Lidia Elizabeth, 2J

Vogelsberg, Ashten, 10

Waibel, Friedrich, $1 \mathrm{~S}$

Wan, Yongjian, $1 Z$

Wang, Jia, $1 \mathrm{Z}$

Waver, Jochen, IT

Whitsitt, Rebecca, OP

Wickenhagen, S., OR, 29

Wilbrandt, Steffen, IT

Wilde, C., OL

Wolfs, Franciscus, 15

Wu, Fan, $1 Y$

Wu, Lunzhe, 04

Xing, Tingwen, 09

$\mathrm{Xu}$, Longbo, $1 \mathrm{~J}$

Xu, Xueke, 04, $1 \mathrm{~J}$

Yang, Minghong, 04

Yang, Suncheol, 2B

Yang, Weiguang, 04

Yu, Zong-Ru, 23

Zhang, Chupeng, $2 Q$

Zhang, Huifang, 04

Zhao, Huiying, 2Q

Zhong, Xianyun, $1 Y$

Zhou, You, $1 \mathrm{~J}$

Zu, Lijun, OE

Proc. of SPIE Vol. 10448 1044801-11

Downloaded From: https://www.spiedigitallibrary.org/conference-proceedings-of-spie on 26 Apr 2023 Terms of Use: https://www.spiedigitallibrary.org/terms-of-use 
Proc. of SPIE Vol. 10448 1044801-12 Downloaded From: https://www.spiedigitallibrary.org/conference-proceedings-of-spie on 26 Apr 2023
Terms of Use: https://www.spiedigitallibrary.org/terms-of-use 


\title{
Conference Committee
}

\author{
Symposium Chairs
}

Julie L. Bentley, University of Rochester (United States)

Sebastian Stoebenau, OptoTech Optikmaschinen GmbH (Germany)

Conference Chair

Julie L. Bentley, University of Rochester (United States)

Conference Co-Chair

Sebastian Stoebenau, OptoTech Optikmaschinen GmbH (Germany)

\section{Conference Program Committee}

Thomas Battley, New York Photonics Industry Association (United States)

Michael J. Bechtold, OptiPro Systems (United States)

Christopher T. Cotton, ASE Sailing Inc. (United States)

Walter C. Czajkowski, Optical Consultant (United States)

Michael A. DeMarco, QED Technologies, Inc. (United States)

Apostolos Deslis, JENOPTIK Optical Systems, LLC (United States)

Toshihide Dohi, OptiWorks, Inc. (Japan)

Dan Gauch, Schneider Optical Machines Inc. (United States)

Tom Godin, Satisloh North America Inc. (United States)

Heidi Hofke, OptoTech Optical Machinery Inc. (United States)

Jay Kumler, JENOPTIK Optical Systems, LLC (United States)

Justin J. Mahanna, Universal Photonics Inc. (United States)

Michael A. Marcus, Lumetrics, Inc. (United States)

Paul Meier-Wang, AccuCoat Inc. (United States)

Ted Mooney, Harris Geospatial Systems (United States)

Michael N. Naselaris, Sydor Optics, Inc. (United States)

Richard Nastasi, Universal Photonics Inc. (United States)

John J. Nemechek, Metrology Concepts LLC (United States)

Buzz Nesti, Naked Optics Corporation (United States)

Matthias Pfaff, OptoTech Optikmaschinen GmbH (Germany)

Paul Tolley, Smart System Technology \& Commercialization Center (United States)

Blair L. Unger, Rochester Precision Optics, LLC (United States)

Martin J. Valente, Arizona Optical Systems, LLC (United States)

Kirk J. Warden, LaCroix Optical Company (United States) 
Robert Wiederhold, Optimax Systems, Inc. (United States)

Dhananjay Joshi, Clemson University (United States)

\section{Session Chairs}

Plenary Session

Julie L. Bentley, University of Rochester (United States)

$1 \quad$ Grinding and Polishing Processes I

Sebastian Stoebenau, OptoTech Optikmaschinen GmbH (Germany)

2 Grinding and Polishing Processes II

Jessica Nelson, Optimax Systems, Inc. (United States)

3 Grinding and Polishing Processes III

John C. Lambropoulos, University of Rochester (United States)

4 Additive Manufacturing

Ulrike Fuchs, asphericon GmbH (Germany)

6 Optical Design and Engineering

Blair L. Unger, Rochester Precision Optics, LLC (United States)

7 Diamond Turning

James T. Mooney, Harris Corporation (United States)

$8 \quad$ Molding

Matthias Pfaff, OptoTech Optikmaschinen GmbH (Germany)

9 Freeform Fabrication and Testing

Kate Medicus, Optimax Systems, Inc. (United States)

10 Metrology

Dan Gauch, Schneider Optical Machines Inc. (United States)

11 Metrology II

Paul E. Murphy, QED Technologies, Inc. (United States)

12 Metrology III

Filipp Ignatonich, Lumetrics, Inc. (United States)

13 Optical Materials

Filipp Ignatonich, Lumetrics, Inc. (United States)

14 Coating and Cleaning

Jennifer D. T. Kruschwitz, University of Rochester, Institute of Optics (United States) 
Proc. of SPIE Vol. 10448 1044801-15

Downloaded From: https://www.spiedigitallibrary.org/conference-proceedings-of-spie on 26 Apr 2023 Terms of Use: https://www.spiedigitallibrary.org/terms-of-use 
Proc. of SPIE Vol. 10448 1044801-16

Downloaded From: https://www.spiedigitallibrary.org/conference-proceedings-of-spie on 26 Apr 2023 Terms of Use: https://www.spiedigitallibrary.org/terms-of-use 